\title{
Patients with pemphigus are at an increased risk of developing rheumatoid arthritis: a large-scale cohort study
}

\author{
Khalaf Kridin ${ }^{1}$ (D) Virginia A. Jones ${ }^{2} \cdot$ Payal M. Patel $^{2} \cdot$ Shira Zelber-Sagi ${ }^{3} \cdot$ Christoph M. Hammers $^{1}$. \\ Giovanni Damiani ${ }^{4} \cdot$ Kyle T. Amber $^{2} \cdot$ Arnon D. Cohen $^{5}$
}

Received: 12 June 2020 / Accepted: 22 October 2020 / Published online: 7 November 2020

(C) The Author(s) 2020

\begin{abstract}
Data regarding the association between pemphigus and rheumatoid arthritis (RA) is inconclusive and yet to be firmly established. In the current study, we aimed to evaluate the risk of developing RA during the course of pemphigus. A large-scale populationbased longitudinal cohort study was conducted to evaluate the hazard ratio (HR) of RA among 1985 patients with pemphigus relative to 9874 age-, sex-, and ethnicity-matched control subjects. A multivariate Cox regression model was utilized. The incidence of RA was 1.07 (95\% CI, 0.62-1.72) and 0.36 (95\% CI, 0.24-0.52) per 1000 person-years among patients with pemphigus and controls, respectively. The lifetime prevalence of RA was $2.3 \%$ (95\% CI, 1.7-3.1\%) among cases and $1.8 \%(95 \%$ CI, $1.5-2.0 \%$ ) among controls. Patients with pemphigus were more than twice as likely to develop RA as compared to control subjects (adjusted HR, 2.54; 95\% confidence interval [CI], 1.31-4.92). The increased risk was robust to a sensitivity analysis that included only cases managed by pemphigus-related systemic medications (adjusted HR, 2.56; 95\% CI, 1.30-5.05). In conclusion, pemphigus is associated with an increased risk of RA. Physicians treating patients with pemphigus should be aware of this possible association. Further research is required to better understand the mechanism underlying this association.
\end{abstract}

Keywords Rheumatoid arthritis $\cdot$ Pemphigus $\cdot$ Cohort study

\section{Introduction}

Pemphigus encompasses a group of rare, potentially lethal, mucocutaneous autoimmune bullous dermatoses [1]. These intraepidermal bullous diseases manifest with vesicles and erosions on the epithelium of mucous membranes and skin, often causing a substantial impairment of quality of life and

Kyle T. Amber and Arnon D. Cohen contributed equally to this work.

Khalaf Kridin

Dr_kridin@hotmail.com; arcohen@clalit.org.il

1 Lübeck Institute of Experimental Dermatology, University of Lübeck, Ratzeburger Allee 160, 23562 Lübeck, Germany

2 Department of Dermatology, University of Illinois at Chicago, Chicago, IL, USA

3 University of Haifa, Haifa, Israel

4 Clinical Dermatology, IRCCS Istituto Ortopedico Galeazzi, 20161 Milan, Italy

5 Clalit Health Services, Tel-Aviv, Israel elevated morbidity and mortality $[2,3]$. The pathogenesis results from the production of IgG autoantibodies against epidermal cadherins $[4,5]$. It is well studied that autoimmune disease, such as pemphigus, is likely to coexist within individuals and their relatives [6-8]. Several studies have substantiated the concept of autoimmune diathesis in pemphigus with an array of autoimmune diseases [1].

Rrheumatoid arthritis (RA) is a chronic, usually symmetric, inflammatory autoimmune disease, primarily afflicting peripheral joints [9]. While the exact etiology of RA is not fully understood, environmental factors such as smoking, in a genetically prone individual, are believed to the major stimuli in RA development [9]. A high burden of autoimmune disease had been observed among patients with RA, with $24 \%$ of patients having at least one concomitant autoimmune disease [10].

A descriptive cluster analysis has demonstrated that pemphigus forms a distinct cluster with (RA autoimmune thyroid diseases (AITD), and type I diabetes mellitus[11]. In the meanwhile, two other observational studies did not reveal a significant association between pemphigus and RA [3, 12]. Of interest, a reciprocal human leukocyte antigen (HLA) disease 
association between the HLA-DRB1 alleles for pemphigus vulgaris $(\mathrm{PV})$ and RA has been established in the literature [13]. While HLA-DRB1*04:02 allele confers a predisposing risk to PV, it is protective in RA $[13,14]$. Taken together, the association between these conditions is inconclusive and yet to be firmly established both epidemiologically and genetically.

The aim of the current study is to further explore the association between pemphigus and RA by conducting a largescale retrospective cohort study investigating the risk of developing RA among patients with pemphigus.

\section{Methods}

\section{Study design and database}

We conducted a retrospective cohort study to gauge the risk of developing incident RA among patients with pemphigus compared to control individuals.

The information for this study was obtained from the computerized dataset of Clalit Healthcare Services (CHS). CHS is the largest Israeli health organization, providing both public and semi-private healthcare services to approximately $4,400,000$ insured patients as of 2016 (which is approximately $54 \%$ of Israel's general population consistent with the 2016 census). CHS allows research professionals to automatically retrieve and extract patient data for epidemiological studies by use of a comprehensive computerized database with constant real-time input from medical, pharmaceutical, and administrative operating systems.

Additionally, CHS offers a chronic disease registry which collects data from different sources, including those of health systems, primary care physicians, and specialists, and are regarded as vastly reliable[15].

\section{Study population and covariates}

Pemphigus and RA disease diagnoses were defined by the following criteria: a documented diagnosis of these entities, twice, as a minimum, in the medical records as documented by a community physician, or when hospital discharge documentation recorded the diagnoses. The control group consisted of up to 5 controls per patient, matched at random by age, gender, and ethnicity. Age matching was based on the exact year of birth (1-year strata). Diagnosis date was used as an index date for the cases and each matched control.

A sensitivity analysis aiming to increase the validity of the diagnosis was performed. The latter repeated all calculations after including only cases prescribed "pemphigus-related medications": systemic corticosteroids or adjuvant immunosuppressive agents (azathioprine, mycophenolate mofetil, or cyclophosphamide) for more than 6 months; or cases prescribed one or more cycles of rituximab.

Outcome measures were controlled for healthcare overutilization in order to rule out ascertainment bias in our observed associations. Healthcare utilization was defined by each individual's total visit number in the year prior to the diagnosis of pemphigus or the date of enrollment for control subjects. Additional adjustment for comorbid conditions was performed by use of the Charlson comorbidity index; a validated method of measuring comorbidity which has been shown to be a dependable predictor of lethal outcomes [16].

\section{Statistical analysis}

Baseline characteristics are described by means and standard deviations (SDs) for continuous variables, whereas frequencies and percentages are used to describe categorical values. A comparison of the distribution of sociodemographic and clinical factors between patients with and without pemphigus was conducted, where the chi-square test was used to describe sex and socioeconomic status, and $t$ test was used for age.

Incidence rates of RA were calculated for both pemphigus patients and controls and expressed as the number of events per 1000 person-years. Hazard ratios (HRs) for the risk of incident RA were obtained by use of Cox regression models, whereas odds ratio (OR) were calculated through logistic regression. The incidence of RA during follow-up was calculated only for individuals without a history of RA before study initiation. The cumulative incidence of RA was compared between pemphigus and control groups using a stratified $\log$-rank test. Two-tailed $P$ values less than 0.05 were considered statistically significant, whereas results with $95 \%$ confidence intervals (CIs) were reported where applicable. SPSS software, version 25 (SPSS, Chicago, IL, USA) was utilized to perform all statistical analyses.

\section{Results}

A total of 1985 patients with pemphigus, diagnosed between 2004 and 2014 and 9874 age-, sex-, and ethnicity-matched control subjects were included in the study. No significant difference was found between the average age with, sex distribution, ethnicity, and socioeconomic status of the two groups; the mean $( \pm \mathrm{SD})$ age of study participants was 72.1 \pm 18.5 years and $40.2 \%$ were males. The Charlson comorbidity index rate was higher in pemphigus patients, with 1059 (53.4\%) patients affected by severe comorbidity compared to 4055 (41.1\%) control subjects $(P<0.001$; Table 1$)$.

The lifetime prevalence of RA was slightly higher among patients with pemphigus $(2.3 \%$; $95 \% \mathrm{CI}, 1.7-3.1 \%)$ as compared to controls $(1.8 \% ; 95 \% \mathrm{CI}, 1.5-2.0 \%)$, although it did not exceed the level of statistical significance (OR, 1.33; $95 \%$ 
Table 1 Descriptive characteristics of the study population

\begin{tabular}{llll}
\hline Characteristic & Patients with pemphigus $(N=1985)$ & Controls $(N=9874)$ & $P$ value \\
\hline Age, years & & & \\
$\quad$ Mean \pm SD & $72.1 \pm 18.5$ & $72.1 \pm 18.5$ & 1.000 \\
Median (range) & $77.4(0-103.0)$ & $77.4(0-103.1)$ & \\
Male sex, $N(\%)$ & $797(40.2 \%)$ & $3962(40.1 \%)$ & 0.934 \\
Ethnicity, $N(\%)$ & & & \\
Jews & $1805(90.9 \%)$ & $8866(89.8 \%)$ & 0.136 \\
Arabs & $180(9.1 \%)$ & $1008(10.2 \%)$ & \\
BMI, kg/m ${ }^{2}(m e a n+S D)$ & $27.7 \pm 6.6$ & $27.9 \pm 6.6$ & 0.355 \\
Smoking, $N(\%)$ & $510(25.7 \%)$ & $2758(27.9 \%)$ & 0.045 \\
SES, $N(\%)$ & & & \\
Low & $634(31.9 \%)$ & $3249(32.9 \%)$ & 0.386 \\
Intermediate & $830(41.8 \%)$ & $4263(43.2 \%)$ & 0.250 \\
High & $423(21.3 \%)$ & $2217(22.5 \%)$ & 0.241 \\
Charlson comorbidity score, $n(\%)$ & & \\
None $(0)$ & $344(17.3 \%)$ & $2636(26.7 \%)$ & $<0.001$ \\
Moderate $(1-2)$ & $582(29.3 \%)$ & $3183(32.2 \%)$ & 0.011 \\
Severe $(\geq 3)$ & $1059(53.4 \%)$ & $4055(41.1 \%)$ & $<0.001$ \\
\hline
\end{tabular}

Abbreviations: $N$, number; $S D$, standard deviation; $B M I$, body mass index; $S E S$, socioeconomic status
CI, 0.96-1.85). In cases where RA preceded the onset of pemphigus, the mean latency from RA to pemphigus was $8.2 \pm 4.0$ years, whereas when pemphigus preceded the onset of RA, the mean latency to RA was $3.4 \pm 3.3$ years.

During the follow-up period, a total of 15 and 25 patients developed new-onset RA, among the case and control groups, respectively. The total follow-up time was $14,046.0$ personyears for patients with pemphigus and 70,378.5 person-years for controls. Taken together, the incidence of RA was 1.07 (95\% CI, 0.62-1.72) and $0.36(95 \% \mathrm{CI}, 0.24-0.52)$ per 1000 person-years among patients with pemphigus and controls, respectively (Table 2).

The crude risk of RA was threefold higher in patients with pemphigus than in control individuals (HR, 3.00; 95\% CI, 1.58-5.69; Fig. 1). In a subgroup analysis, the crude risk was increased in female (HR, 3.34; 95\% CI, 1.70-6.58), but not in male (HR, 1.25; 95\% CI, 0.14-11.17) patients. After adjusting for several confounding factors, including demographic features, healthcare utilization, and comorbidities, pemphigus remained an independent significant risk factor
Table 2 Incidence rates and hazard ratio of new-onset rheumatoid arthritis among patients with pemphigus (cohort study design)

\begin{tabular}{lll}
\hline & Patients with pemphigus & Controls \\
\hline Follow-up time, PY & $14,046.0$ & $70,378.5$ \\
Median follow-up time, years (range) & $6.9(0.0-13.0)$ & $6.9(0.0-13.0)$ \\
Number of events & 15 & 25 \\
Incidence rate/1000 PY & 1.07 & 0.36 \\
$95 \%$ CI & $0.62-1.72$ & $0.24-0.52$ \\
& HR $(95 \% \mathrm{CI})$ & $P$ value \\
Crude & $3.00(1.58-5.69)$ & 0.001 \\
Adjusted* & $2.54(1.3-4.92)$ & 0.006 \\
Sensitivity analysis** & & $<0.001$ \\
Crude & $3.30(1.70-6.45)$ & 0.007 \\
Adjusted* & $2.56(1.30-5.05)$ & \\
\hline
\end{tabular}

Abbreviations: $H R$, hazard ratio; $C I$, confidence interval; $P Y$, person-year

*Following the adjustment for age, sex, ethnicity, socioeconomic status, comorbidities, and healthcare utilization **Sensitivity analysis included only pemphigus patients under prolonged "pemphigus-specific treatments" Italic: significant value 
Fig. 1 The cumulative incidence of RA among patients with pemphigus and controls

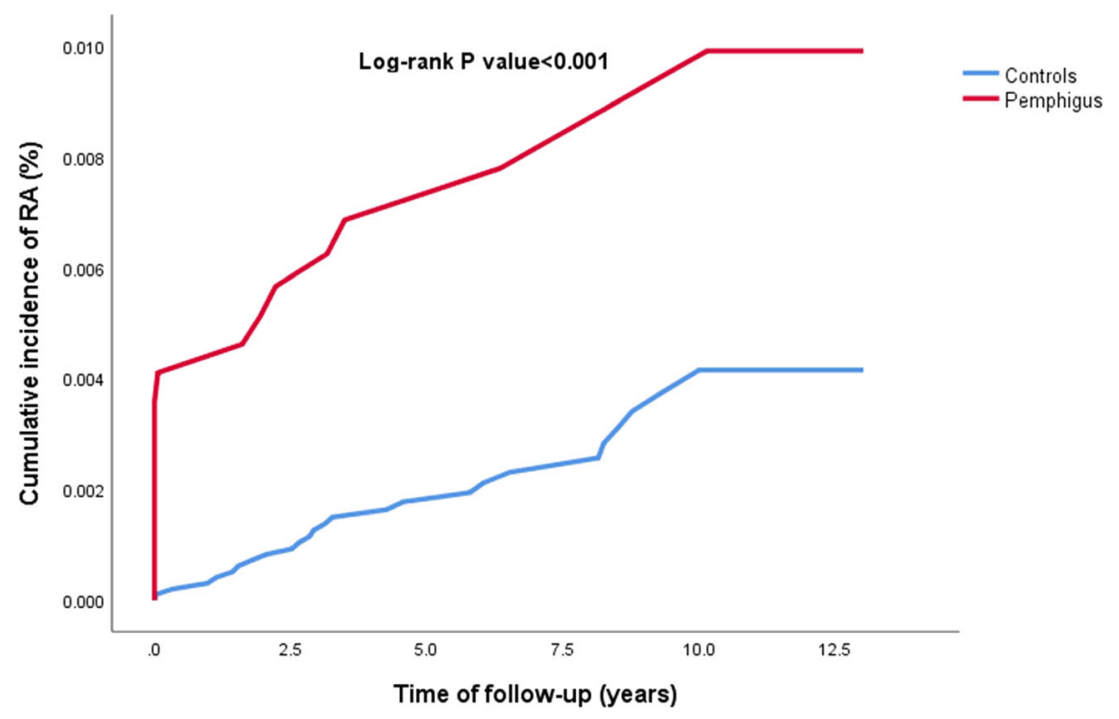

for incident RA (adjusted HR, 2.54; 95\% CI, 1.31-4.92; Table 2).

We then conducted a sensitivity analysis including solely patients with pemphigus who were prescribed "pemphigusrelated treatments." The crude risk of RA had been substantiated (HR, 3.30; 95\% CI, 1.70-6.45), whereas the adjusted risk was not altered substantially following the adjustment for several putative confounding factors (adjusted HR, 2.56; 95\% CI, 1.30-5.05; Table 2)

Of note, a diagnosis of both pemphigus and RA does not significantly increase the risk of all-cause mortality relative to the mortality observed among patients with isolated pemphigus (HR, 1.31; 95\% CI, 0.91-1.87).

\section{Discussion}

The current retrospective cohort study demonstrates that pemphigus patients are at more than twofold increased risk of developing RA as compared to control subjects (HR, 2.54). The results revealed that the risk of RA was particularly elevated in females (HR, 3.34), a finding that accords with the female preponderance observed in several autoimmune diseases [17, 18].

\section{The association between pemphigus and RA in the previous literature}

Based on the findings of well-designed epidemiological studies, pemphigus has been reported to associate with other autoinflammatory and autoimmune comorbid disorders, such as RA [11, 19], ulcerative colitis [20], AITD [11, 19, 21], and psoriasis [3, 12, 22, 23]. Likewise, patients with RA experience a high burden of comorbid autoimmune conditions. A large-scale cross-sectional study had found that $24.3 \%$ and $6.0 \%$ of patients with RA have at least one and more than one comorbid autoimmune disease, respectively, with ankylosing spondylitis and psoriatic arthritis being most frequently encountered [10]. These observations are perceived as part of the concept known as "autoimmune diathesis," in which individuals affected by an autoimmune disease are more vulnerable to acquire other autoimmune disease [6-8].

Leshem et al. [19] and Parameswaran et al. [11] revealed that the prevalence of RA was significantly greater among patients with pemphigus than in their first-degree relatives and the general population, respectively. Both studies, however, were uncontrolled and small scale, and the latter study relied on surveys and patient-reported outcomes. In contrast, a case-control study from Taiwan [12] and a cross-sectional study from the USA [3] failed to demonstrate a significant association between the two conditions. While the association between pemphigus and RA had been evaluated in the aforementioned studies [3, 11, 12, 19], the incidence of RA among patients with pemphigus, as well as the risk of RA during the course of pemphigus, is yet to be investigated.

\section{Putative interpretation for the study findings}

The mechanism responsible for the association between RA and pemphigus is yet to be determined, but immunogenetic involvement has been speculated. Immunogenetic studies show an important association between susceptibility to PV and $H L A-D R B 1 * 04: 02$, primarily in Ashkenazi Jews, and $H L A-D R B 1 * 14: 01, H L A-D R B 1 * 14: 04$, and HLA$D Q B 1 * 05: 03$, prevalent in non-Jewish patients of European and Asian descent [24]. Yet, $H L A-D R B 1 * 04: 02$ is protective in RA, as it lacks the "shared epitope" seen in several high-risk HLA allotypes in RA. Notably, this shared epitope is also missing from DRB1*13:01, DRB1*13:02 [13]; thus, HLA $D R B 1 * 04: 02$ does not appear to be a shared risk factor in our population. We hypothesize that alternative $\mathrm{T}$ cell 
regulatory pathways may explain this shared autoimmune phenomenon in the presence of contradictory data for $D R B 1 * 04: 02$ in both diseases [25].

Interleukin 17 (IL-17) is a pro-inflammatory cytokine produced by Th17 cells which serves as a bridge between innate and adaptive immunity [26, 27]. Evidence supports an association between RA and IL-17 elucidating that excessive IL17 receptor signaling is an essential pathway in converting an acute synovitis into the chronically damaging arthritis seen in RA [26-28]. Likewise, a recent meta-analysis has identified vastly increased levels of serum IL-17 in patients with pemphigus, perhaps expounding on the association between pemphigus and RA [29].

\section{Strengths and limitations}

While the current study is the first cohort study to shed light on the association between pemphigus and RA, and to contributes a novel epidemiological feature regarding the risk of developing RA during the course of pemphigus, it is not without limitations. The management of pemphigus patients generally includes high doses of corticosteroids and to immunosuppressive agents [30-32], which should theoretically improve subclinical RA or mask any underlying symptoms [33]. We speculate that this may lead to an underestimation in the incidence of RA among patients with pemphigus due to underreported diagnosis of RA. The diagnosis of cases with pemphigus and RA was not made on the basis of histologic or immunopathological criteria, and the clinical characteristics of eligible cases could not be retrieved. However, previous studies had demonstrated that the chronic registry of CHS was proven very reliable.

In conclusion, we demonstrate an increased risk of developing RA among female pemphigus patients. Prospective studies are needed to evaluate this epidemiological relationship in other ethnic groups. In pemphigus patients presenting with concerning symptoms of RA, additional investigation may be warranted.

Funding Open Access funding enabled and organized by Projekt DEAL.

\section{Compliance with ethical standards}

IRB approval status Exempt.

Conflict of interest The authors declare no conflict of interests.

Open Access This article is licensed under a Creative Commons Attribution 4.0 International License, which permits use, sharing, adaptation, distribution and reproduction in any medium or format, as long as you give appropriate credit to the original author(s) and the source, provide a link to the Creative Commons licence, and indicate if changes were made. The images or other third party material in this article are included in the article's Creative Commons licence, unless indicated otherwise in a credit line to the material. If material is not included in the article's
Creative Commons licence and your intended use is not permitted by statutory regulation or exceeds the permitted use, you will need to obtain permission directly from the copyright holder. To view a copy of this licence, visit http://creativecommons.org/licenses/by/4.0/.

\section{References}

1. Kridin K. Pemphigus group: overview, epidemiology, mortality, and comorbidities. Immunol Res. 2018 [cited 2018 Mar 24]; Available from: http://link.springer.com/10.1007/s12026-0188986-7

2. Kridin K, Sagi S, Bergman R. Mortality and cause of death in Israeli patients with pemphigus. Acta Derm Venereol. 2017 [cited 2017 Mar 9];0. Available from: http://www.ncbi.nlm.nih.gov/ pubmed/28093595

3. Hsu DY, Brieva J, Sinha AA, Langan SM, Silverberg JI. Comorbidities and inpatient mortality for pemphigus in the U.S.A. Br J Dermatol. 2016;174:1290-8.

4. Amber KT, Valdebran M, Grando SA. Non-desmoglein antibodies in patients with pemphigus vulgaris. Front Immunol. 2018;4(9): 1190. https://doi.org/10.3389/fimmu.2018.01190.

5. Hammers CM, Stanley JR. Mechanisms of disease: pemphigus and bullous pemphigoid. Annu Rev Pathol Mech Dis. 2016;11:175-97 Available from: http://www.annualreviews.org/doi/10.1146/ annurev-pathol-012615-044313.

6. Somers EC, Thomas SL, Smeeth L, Hall AJ. Autoimmune diseases co-occurring within individuals and within families: a systematic review. Epidemiology. 2006;17:202-17.

7. Szyper-Kravitz M, Marai I, Shoenfeld Y. Coexistence of thyroid autoimmunity with other autoimmune diseases: friend or foe? Additional aspects on the mosaic of autoimmunity. Autoimmunity. 2005;38:247-55.

8. Davidson A, Diamond B. Autoimmune diseases. N Engl J Med. 2001;345:340-50 Available from: http://www.ncbi.nlm.nih.gov/ pubmed/11484692.

9. Calabresi E, Petrelli F, Bonifacio AF, Puxeddu I, Alunno A. One year in review 2018: pathogenesis of rheumatoid arthritis. Clin Exp Rheumatol. 2018;36(2):175-84.

10. Simon TA, Kawabata H, Ray N, Baheti A, Suissa S, Esdaile JM. Prevalence of co-existing autoimmune disease in rheumatoid arthritis: a cross-sectional study. Adv Ther. 2017;34:2481-90.

11. Parameswaran A, Attwood K, Sato R, Seiffert-Sinha K, Sinha AA. Identification of a new disease cluster of pemphigus vulgaris with autoimmune thyroid disease, rheumatoid arthritis and type I diabetes. Br J Dermatol. 2015;172:729-38.

12. Chiu Y-W, Chen Y-D, Hua T-C, Wu C-H, Liu H-N, Chang Y-T. Comorbid autoimmune diseases in patients with pemphigus: a nationwide case-control study in Taiwan. Eur J Dermatol. 2017 [cited 2017 Dec 2];27:375-81. Available from: http://www.ncbi.nlm.nih. gov/pubmed/28747284.

13. Van Drongelen V, Holoshitz J. A reciprocal HLA-disease association in rheumatoid arthritis and pemphigus vulgaris. Front Biosci Landmark. 2017;1(22):909-19. https://doi.org/10.2741/4524.

14. Amber KT, Staropoli P, Shiman MI, Elgart GW, Hertl M. Autoreactive $\mathrm{T}$ cells in the immune pathogenesis of pemphigus vulgaris. Exp Dermatol. 2013;22:699-704.

15. Rennert G, Peterburg Y. Prevalence of selected chronic diseases in Israel. Isr Med Assoc J. 2001 [cited 2017 Jan 30];3:404-8. Available from: http://www.ncbi.nlm.nih.gov/pubmed/11433630

16. Charlson ME, Pompei P, Ales KL, MacKenzie CR. A new method of classifying prognostic comorbidity in longitudinal studies: 
development and validation. J Chronic Dis. 1987;40:373-83 Available from: http://www.ncbi.nlm.nih.gov/pubmed/3558716.

17. Dooley MA, Hogan SL. Environmental epidemiology and risk factors for autoimmune disease. Curr Opin Rheumatol. 2003;15:99103.

18. Jacobson DL, Gange SJ, Rose NR, Graham NMH. Epidemiology and estimated population burden of selected autoimmune diseases in the United States. Clin Immunol Immunopathol. 1997;84(3): 223-43. https://doi.org/10.1006/clin.1997.4412.

19. Leshem YA, Katzenelson V, Yosipovitch G, David M, Mimouni D. Autoimmune diseases in patients with pemphigus and their firstdegree relatives. Int J Dermatol. 2011;50:827-31.

20. Kridin K, Zelber-Sagi S, Comaneshter D, Cohen AD. Ulcerative colitis associated with pemphigus: a population-based large-scale study. Scand J Gastroenterol. 2017;52:1360-4.

21. Kridin K, Khamaisi M, Comaneshter D, Batat E, Cohen AD. Autoimmune thyroid diseases and thyroid cancer in pemphigus: a big data analysis. Front Med. Frontiers; 2018 [cited 2018 Jun 7];5: 159. Available from: https://www.frontiersin.org/article/10.3389/ fmed.2018.00159/full

22. Kridin K, Zelber-Sagi S, Comaneshter D, Cohen AD. Association between pemphigus and psoriasis: A population-based large-scale study. J Am Acad Dermatol. 2017 [cited 2017 Dec 2];77:1174-5. Available from: http://www.ncbi.nlm.nih.gov/pubmed/29132851

23. Kridin K, Kridin M, Shalom G, Cohen AD. The coexistence of pemphigus and psoriasis: a systematic review and meta-analysis. Immunol Res. 2018 [cited 2019 Feb 18]; Available from: http:/ link.springer.com/10.1007/s12026-018-9031-6

24. Yan L, Wang JM, Zeng K. Association between HLA-DRB1 polymorphisms and pemphigus vulgaris: A meta-analysis. Br J Dermatol. 2012;167:768-77.

25. Scheinecker C, Göschl L, Bonelli M. Treg cells in health and autoimmune diseases: new insights from single cell analysis. J Autoimmun. 2019.
26. Char JJJ, Jaller JA, Raad JJJ, Waibel JS, Bhanusali DG, Bhanusali $\mathrm{N}$. The role of IL-17 in the human immune system and its blockage as a treatment of rheumatoid arthritis, ankylosing spondylitis, and psoriatic arthritis. J Drugs Dermatol. 2018.

27. Bunte K, Beikler T. Th17 cells and the IL-23/IL-17 axis in the pathogenesis of periodontitis and immune-mediated inflammatory diseases. Int J Mol Sci. 2019;20.

28. Lubberts E. IL-17/Th17 targeting: on the road to prevent chronic destructive arthritis? Cytokine. 2008;41(2):84-91. https://doi.org/ 10.1016/j.cyto.2007.09.014.

29. Kowalski EH, Kneibner D, Kridin K, Amber KT. Serum and blister fluid levels of cytokines and chemokines in pemphigus and bullous pemphigoid. Autoimmun Rev. 2019 [cited 2019 Mar 12]; Available from: http://www.ncbi.nlm.nih.gov/pubmed/30844553

30. Amber KT, Maglie R, Solimani F, Eming R, Hertl M. Targeted therapies for autoimmune bullous diseases: current status. Drugs. 2018;78:1527-48.

31. Kridin K, Ahn C, Huang WC, Ansari A, Sami N. Treatment update of autoimmune blistering diseases. Dermatol Clin. 2019 [cited 2019 Mar 12];37:215-28. Available from: http://www.ncbi.nlm.nih.gov/ pubmed/30850044

32. Kridin K. Emerging treatment options for the management of pemphigus vulgaris. Ther Clin Risk Manag. 2018 [cited 2018 May 13];Volume 14:757-78. Available from: http:/www.ncbi. nlm.nih.gov/pubmed/29740210

33. Pincus T, O'Dell JR, Kremer JM. Combination therapy with multiple disease-modifying antirheumatic drugs in rheumatoid arthritis: A preventive strategy. Ann Intern Med. 1999;131:768.

Publisher's note Springer Nature remains neutral with regard to jurisdictional claims in published maps and institutional affiliations. 